\title{
Blood pressure management and regional cerebral oxygen saturation during surgery in beach chair position
}

It was interesting to read the paper by Ko et al. [1] regarding cerebral oxygen saturation monitoring in patients undergoing an arthroscopic shoulder surgery in beach chair position (BCP). While mostly agreeing with their conclusion, one may also raise a few concerns, such as the timely determination of baseline values and the proper use of vasopressors.

As to whether blood pressure should be corrected to account for the level difference between the site of measurement (most commonly a non-invasive cuff on the non-operative arm) and the brain in BCP is under debate [2]. They indeed evaluated whether MAP measured at the level of the brain or at the level of the heart correlated with regional cerebral oxygen saturation $\left(\mathrm{rSO}_{2}\right)$ in patients undergoing BCP surgery, and found that MAP measured at the brain, but not at the heart level, correlated with $\mathrm{rSO}_{2}$. Nevertheless, they stated in the conclusion that "Monitoring cerebral $\mathrm{rSO}_{2}$ and MAP at the level of brain can be helpful to detect the possibility of cerebral deoxygenation earlier" [1]. To admit their observation, it would be proper to state that "MAP measured at the brain but not at the heart level reflects $\mathrm{rSO}_{2}$ in patients undergoing surgery in BCP".

They also mentioned that "Baseline mean arterial pressure, ---, BIS, and left and right $\mathrm{rSO}_{2}$ were recorded while the patient's condition was stable over 5 minutes before induction." However, the induction of anesthesia and the use of high inspired oxygen fraction per se may significantly decrease BIS and increase $\mathrm{rSO}_{2}$ values [3]. As such, we believe that these parameters measured after (i.e., immediately before patients were raised into BCP) the induction of anesthesia better represents the baseline values.

Phenylephrine, an $\alpha$-agonist, and ephedrine, an $\alpha$ - and $\beta$ agonist, are both commonly used to maintain MAP and thus, cerebral perfusion pressure in neurosurgical patients who develop hypotension. However, in healthy anesthetized subjects with intact cerebral autoregulation, a bolus administration of phenylephrine reduced the $\mathrm{rSO}_{2}$ measured by a nearinfrared spectroscopy device under intravenous anesthesia with propofol/remifentanil, whereas ephedrine maintained it $[4,5]$. In this context, phenylephrine and ephedrine may have differentially affected $\mathrm{rSO}_{2}$ in their study. Moreover, they did not use equipotent dosages of the two vasopressors. Would it not be better for them to have included it as one of the limitations of the study?

Dong Ho Kang, and Kyung Yeon Yoo

Department of Anesthesiology and Pain Medicine, Chonnam National University Hospital, Gwangju, Korea

\section{Kyung Yeon Yoo}

Department of Anesthesiology and Pain Medicine, Chonnam National University Hospital, Gwangju, Korea

E-mail: kyyoo@jnu.ac.kr

\section{References}

1. Ko SH, Cho YW, Park SH, Jeong JG, Shin SM, Kang G. Cerebral oxygenation monitoring of patients during arthroscopic shoulder surgery in the sitting position. Korean J Anesthesiol 2012; 63: 297 301.

2. Murphy GS, Szokol JW. Blood pressure management during beach chair position shoulder surgery: what do we know? Can J Anaesth 2011; 58: 977-82.

3. Lovell AT, Owen-Reece H, Elwell CE, Smith M, Goldstone JC. Continuous measurement of cerebral oxygenation by near infrared spectroscopy during induction of anesthesia. Anesth Analg 1999; 88: 554-8.

4. Meng L, Cannesson M, Alexander BS, Yu Z, Kain ZN, Cerussi AE, et al. Effect of phenylephrine and ephedrine bolus treatment on cerebral oxygenation in anaesthetized patients. Br J Anaesth 2011; 107: 209-17.

5. Jeong H, Jeong S, Lim HJ, Lee J, Yoo KY. Cerebral oxygen saturation measured by near-infrared spectroscopy and jugular venous bulb oxygen saturation during arthroscopic shoulder surgery in beach chair position under sevoflurane-nitrous oxide or propofolremifentanil anesthesia. Anesthesiology 2012; 116: 1047-56.

@ This is an open-access article distributed under the terms of the Creative Commons Attribution Non-Commercial License (http:// creativecommons.org/licenses/by-nc/3.0/), which permits unrestricted non-commercial use, distribution, and reproduction in any medium, provided the original work is properly cited. 


\section{In Response}

We thank the opinion writers for their thoughtful comments on our study [1] and are happy to respond to their questions and comments.

We agree with them that the expression "reflects" in the conclusion is much clearer. However, we would like to use a more recommendable expression, such as "be helpful." As you know, we described it because the cerebral $\mathrm{rSO}_{2}$ measured by a near-infrared spectroscopy reflects cerebral oxygen saturation only in the frontal cortex of brain. Moreover, there does not seem to be much difference between their expression and ours.

Also, we used the expression, "baseline mean arterial pressure, heart rate, $\mathrm{SpO}_{2}, \mathrm{BIS}$, and left and right $\mathrm{rSO}_{2}$ ", in the materials and methods. Those parameters were recorded 5 minutes after patient's arrival in the operating room as a reference values and described at the table with demographic data. We agree with questioner's opinion that the values of BIS and $\mathrm{rSO}_{2}$ at the same $\mathrm{FiO}_{2}$ should be used as baseline value. Actually, we used data T0 as baseline value in our study. Data of T0 were collected 5 minutes after intubation and before sitting position $\left(\mathrm{FiO}_{2}\right.$ 0.5) and the same $\mathrm{FiO}_{2}$ was kept throughout the operation [1]. I think that expression made many readers confused.

Evers and Maze describe that phenylephrine administered intravenously to counteract hypotension increases coronary and cerebral perfusion pressure [2]. Hence, it is so interesting to us that a bolus dose of phenylephrine $(100-200 \mu \mathrm{g})$ reduced the cardiac output and $\mathrm{rSO}_{2}$ by NIRS under intravenous anesthesia with propofol/remifentanil in the supine position [3]. The difference with Meng et al. [3] is that we used phenylephrine under general anesthesia with sevoflurane/nitrous oxide in the seated patients. The effect of phenylephrine under inhalational general anesthesia should be considered and compared in the future. We believe that more work is essential.

We appreciate the opinion writers for their thoughtful and informative opinion again. This letter serves to remind all of us that there should be a consideration for using vasoconstrictors in order to keep cerebral perfusion. Further studies are needed to attain more exact information in specific situations and to safely anesthetize patients under a seated position.

Sang-Hun $\mathrm{Ko}^{2}$, Young Woo Cho ${ }^{1}$, Se Hun Park ${ }^{1}$, Jin-Gyu Jeong ${ }^{1}$, Seung-Myeong Shin ${ }^{2}$, and Gun Kang ${ }^{1}$

Departments of ${ }^{1}$ Anesthesiology and Pain Medicine, ${ }^{2}$ Orthopedic Surgery, Ulsan University Hospital, College of Medicine, University of Ulsan, Ulsan, Korea

\section{Se Hun Park}

Department of Anesthesiology and Pain Medicine, Ulsan University Hospital, College of Medicine, University of Ulsan, Ulsan, Korea

E-mail: nurunbab@hotmail.com

\section{References}

1. Ko SH, Cho YW, Park SH, Jeong JK, Shin SM, Kang G. Cerebral oxygenation monitoring of patients during arthroscopic shoulder surgery in the sitting position. Korean J Anesthesiol 2012; 63: 297301.

2. Evers AS, Maze M. Anesthetic pharmacology: physiologic principles and clinical practice. Philadelphia, Churchill Livingstone. 2004, p 602.

3. Meng L, Cannesson M, Alexander BS, Yu Z, Kain ZN, Cerussi AE, et al. Effect of phenylephrine and ephedrine bolus treatment on cerebral oxygenation in anaesthetized patients. Br J Anaesth 2011; 107: 209-17. 